A. Ghorbannia Delavar, S. Hoseyny, R. Maghsoudi / TJMCS Vol .5 No. 2 (2012) 105-114

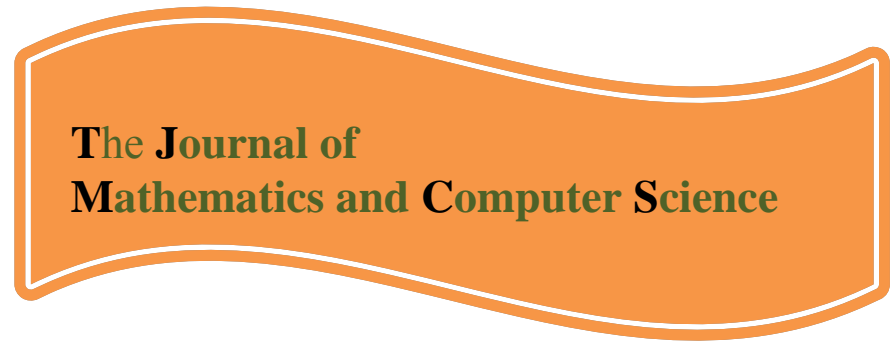

Available online at

http://www.TIMCS.com

The Journal of Mathematics and Computer Science Vol .5 No.2 (2012) 105-114

\title{
BCO-Based Optimized Heuristic Strategies for QoS Routing
}

\author{
Arash Ghorbannia Delavar \\ Assistant Professor, Department of Computer Engineering and Information Technology, \\ Payam Noor University, PO BOX 19395-3697, Tehran, IRAN, \\ a_ghorbannia@pnu.ac.ir \\ Somayyeh Hoseyny (Corresponding Author) \\ Master's Degree Student, Department of Computer Engineering and Information Technology, \\ Payam Noor University, Tehran, IRAN, \\ sce.hoseyny@gmail.com \\ Rouhollah Maghsoudi \\ Department of Computer, Islamic Azad University, Mahmoudabad Branch, Mahmoudabad, Iran, \\ rcemaghsoudi@iaumah.ac.ir
}

Received: February 2012, Revised: November 2012

Online Publication: December 2012

\begin{abstract}
Obtaining an optimized rout such that satisfies Quality factors of Service is a main problem in scope of optimum routings. The search of route that satisfies such multi-constraints as delay, jitter, cost and bandwidth in network can facilitate the solution to multi-media transmission. In this paper, we present a new intelligent routing algorithm QOS using swarm intelligence strategy of bee colony. Swarm intelligence is a relatively novel field. It addresses the study of the collective behaviors of systems made by many components that coordinate using decentralized controls and self-organization. In order to evaluate our strategy, simulation performed under coverage of one of current services of multimedia applications, Video Conference by means of Powerful Simulator of OPNET. Then by MATLAB software, we compared efficiency function of proposed method based on honey bee with genetic algorithm, other current heuristics in QOS. So the strength and accuracy of our method using performed simulations is clear.
\end{abstract}

Keywords: Swarm Intelligence, Bee Colony Optimization, QOS Routing.

\section{Introduction.}

Recently there are many developments occurred in switch and media technologies. This progress is led to appear new generation of high - speed extensive networks. These networks support wide range of real time multimedia applications such in this field which is Quality of Service (QOS) in networks especially multimedia applications. Technically, it is important to deliver 
the information to the receiver on - time, with good quality, without vibration and etc. on - time and real time of multimedia data are often important. For example, in Video - On - Demand (VOD) applications, transfer of the first video frame of transmitter (source) shall not be delayed more than a certain amount, otherwise the user feels that there is a waiting process to receive video. Consequently, it could be expressed that QOS - based routing is one of the important research fields in computer networks. QOS essentials are various in multimedia applications. For example, during routing process, when multimedia data flow is transferring in video conference application, each link must be guaranteed the minimum existing bandwidth. Point to point delay is one of the other important parameters of QOS which is used to assure that sending data by source can arrive to destination in a certain time range. For example, in an online game, users shall be received the sound and new images within a short time and they cannot bear even a few second delay.

Basically, routing is divided into several categories: single - path and multi - path routing, source routing and next step, hierarchical and flat routing, centralized and distributed routing, data - centric and address - centric routing, QOS - based and best - effort routing, event - driven and queue - based routing and energy - based routing. We concentrate on QOS routing in this paper. Many articles has researched this issue and used several methods which are often heuristic and articles intelligence tools. Genetic Algorithm is one of the most famous methods. A fuzzy genetic algorithm is used for QOS routing in article [1]. Exact Information protection of global network status is impossible for nodes of a real dynamic network. Therefore, at first QOS parameters were fuzzed and then using genetic algorithm became fuzzed in order to optimize fitness function. In articles [2 \& 5] a multiple QOS routing algorithm based on genetic algorithm is introduced. In this article, adjacency matrix was used to genotype representation. Some new heuristics were also used to create initial population and cutting and mutation operators. These researchers were looked for a multiple routing tree with the most appropriate QOS parameters.

Evolutionary Optimization strategy is one of the other intelligent methods which is used in QOS multiple routing. In article [3], an evolutionary multi - purpose quick method was proposed as MOEAQ to find the optimized path of QOS; it was better than its basic version for its convergence and in addition to high diversity it could obtain more favorable result compared with a famous routing algorithm based on GA. QOS routing could be performed by comparing several intelligent methods. In article [4], three routing methods were presented based on three intelligent computational methods of genetic algorithm, stimulation annealing and tabu research. These methods were examined on small - scale real networks and some large - scale networks and obtained a good success rate.

Optimization algorithms using in the mentioned research are parts of random optimization algorithms called as Evolutionary Optimization. But also there is the other groups of optimization algorithms which might be included a wider range of application and they are swarm intelligence algorithms, which were used in many of optimization problems such as QOS optimized route. Swarm intelligence is a new and modern field which was inspired from swarm behavior of creatures. A wide range of research in swarm intelligence is performed on reverse engineering and matching collective behaviors seen in natural systems with the goal of effective algorithms design for distributed optimization. These algorithms as inspired from natural systems also have favorable features such as adaptive, scalable and robust. These are key features in routing problem in network. Being adaptive against environmental changes, robustness of loss of population individuals and complete distribution in network. In article [7] an interesting review is performed on application of swarm intelligence algorithms in sensor networks routing. Important approaches of using swarm intelligence in network routing protocols were explained in this article.

In a separate chapter, teamwork mechanisms in ant and bee colonies were investigated and it is surveyed that how these methods are being reverse engineering and used in routing protocols design in different networks. That is why in the last decade researchers were concentrated on routing by basic concepts of swarm intelligence especially Artificial Colony Optimization (ACO) and recently Bee Colony Optimization (BCO).

More accurate investigation of articles shows that researchers were concentrated on using ACO in network routing more and used BCO less [8-9-10-11-12]. Maybe it is because of the youth of BCO. For example, in order to QOS routing, an ACO - based routing algorithm is proposed in article [6] with some limits. Delay, jitter and bandwidth are the limits of this article and they look 
for a route which could be satisfied these multiple limits to simplify a multimedia transfer problem in Ad - hoc with ACO.

We still know that QOS multi - limits problem is a NP - HARD problem. In article [3] by adding a heuristic factor to current ACO, were able the ant not only to use previous findings, but also decrease the effect of pheromone misdirection in unrelated routing and overcome low - speed convergence. As mentioned, using BCO in routing protocol design is an almost new issue and not much works were performed on. For example, articles [13 \& 20] have used BCO to solve colporteur problem which is the most classic and basic problem to find the shortest and best tour in graph and it is a routing issue. In articles [14 \& 15] also BCO has used to routing. In this paper, we proposed an intelligent algorithm using BCO to QOS - based routing, which is not only used network sources with optimized cost but also satisfied the QOS essentials for multimedia applications. However the optimization algorithms such as GA, ACO and ABC might be similar including distributed food collection, population individuals with limited abilities, indirect relations between individuals, etc.

but indeed they are different. For example, ant and bee are two different colonies naturally and behaviorally. Ants walk whether bees fly [7], this difference makes ABC as a search strategy with high - diversity, the point which might be a problem for optimization and evolutionary strategies. Or the foraging process of these two is completely different. The bees left the hive completely to find a new food source; they go far away and after finding a proper source, share it in hive. There are three key abilities in Bee colony which will be very useful in new protocol design: adaptive division for labor, recruitment and communication, stochastic site selection, which all explained in chapter 3.

The other chapters in this paper are classified as follows: Chapter 2 Problem Description and Formulation; Chapter 3 Artificial Bee Colony Algorithm; Chapter 4 Proposed Routing Algorithm; Chapter 5 Evaluation of Proposed Algorithm Efficiency and Comparing with Other Similar Strategies and finally Chapter 6 Conclusion of the Present Research and Future Offers.

\section{Formulation and Problem Description}

A network is explained as connected directed graph $\mathrm{G}=\langle\mathrm{V}, \mathrm{E}\rangle$. V indicates vertices (network's nodes) and E indicates edges (network's links), which shows the connection of vertices. Each link is in mutual nodes, it means that if there is $e=\langle u, v\rangle$ link, there is also $e^{\prime}=\langle v$, $u\rangle$ link. The jitter (e), delay (e), cost (e), loss (e) and $\tau p$ (e) functions, which show QOS essentials to each e link evaluation, are used. Cost function, cost (e), is total distribution, transfer, queuing, rating and switching delays. The loss (e) function is the lost percentage of link e. The jitter (e) and $\tau_{p}$ (e) functions are delayed variance in time range of package arrival and process time in link e, respectively.

However the amount of these functions might be different in sweep status of them, but we assume that cost $(\mathrm{e})=\operatorname{cost}\left(\mathrm{e}^{\prime}\right)$, jitter $(\mathrm{e})=\operatorname{jitter}\left(\mathrm{e}^{\prime}\right)$, delay $(\mathrm{e})=\operatorname{delay}\left(\mathrm{e}^{\prime}\right)$, loss $(\mathrm{e})=\operatorname{loss}\left(\mathrm{e}^{\prime}\right)$ and $\tau_{\mathrm{p}}$ $(\mathrm{e})=\tau_{\mathrm{p}}\left(\mathrm{e}^{\prime}\right)$ in order to simplification. Also we know that the goal is to find an optimized route of above limits from source to destination. We assume this route as $P(s, d)$, in which $s$ is initial node and $\mathrm{d}$ is the last node. Now five criteria of QOS for route $\mathrm{P}$ are definable as follows:

$$
\begin{aligned}
& \operatorname{delay}(\mathrm{P})=\sum_{\mathrm{e} \in \mathrm{P}} \operatorname{delay}(\mathrm{e}) \\
& \operatorname{cost}(\mathrm{P})=\sum_{\mathrm{e} \in \mathrm{P}} \operatorname{cost}(\mathrm{e}) \\
& \operatorname{jitter}(\mathrm{P})=\sum_{\mathrm{e} \in \mathrm{P}} \operatorname{jitter}(\mathrm{e}) \\
& \tau \mathrm{p}(\mathrm{P})=\sum_{\mathrm{e} \in \mathrm{P}} \tau \mathrm{p}(\mathrm{e}) \\
& \operatorname{loss}(\mathrm{P})=1-\prod_{\mathrm{e} \in \mathrm{P}}(1-\operatorname{loss}(\mathrm{P}))
\end{aligned}
$$




\section{Bee Colony Optimization Algorithm}

Food explorer is dived into three categories: Employed Bees, Onlooker Bees and Scout Bees. All bees who are exploiting from a food source are Employed bees. They find the food source, transfer the information of that food source to the hive and share it with Onlooker for new food sources next to the hive. Employed bees share the information of food source by dancing in a determined area inside the hive calling dance zone. This waggle dance depends on nectar content of a food source which is exploiting by dancer bees. The onlooker bees watch the dance and select a food source based on probable relevance with quality of that food source. After selecting the food source, onlooker and scout bees become employed bees. Therefore, good food sources absorb more onlooker bees comparing with bad food sources. When a food source exploited completely, all employed bees left there and might be become onlooker or scout bees. Scout bees could be considered as operators of exploring, while onlooker and employed bees are considered as operators of exploiting [16, 17 and 18]. In ABC algorithm (Karaboga, Basturk, 2008) each food source is a possible answer to the problem and the amount of nectar existing in each food source indicates the quality of the answer which is shown by competency. At first, half of bees consider as employed and the other half as onlooker. Number of food sources are as same as number of employed bees and there is an employed bees per a food source, exactly. Employed bees that left the food source became employed bees again. This algorithm starts with allocation of food source (answer) which produced random to employed bees. In each repetition, each employed bee determines food source near current source and evaluates its nectar (competency). Situation of food source $I$ is indicates as $X_{i}=\left(x_{i 1}, X_{i 2} \ldots X_{i d}\right) . F\left(X_{i}\right)$ indicates the amount of nectar existing in food source which is located in $\mathrm{X}_{\mathrm{i}}$. After watching dance of employed bees, an onlooker bee goes to food source located in $\mathrm{X}_{\mathrm{i}}$ with probability of $\mathrm{P}_{\mathrm{i}}$.

$$
P_{i}=\frac{F(X i)}{\sum_{K=1}^{S} F(X k)}
$$

$\mathrm{S}$ is number of food sources, employed bees or candidate answers.

If the competency of new source is better than the best obtained competency so far (better than competency of the last source), therefore bees go to the new food source and left the previous one, otherwise they remain in the previous food source. When all employed bees finish this process, they share their competency information with onlooker bees and each onlooker bee select a food source based on above mentioned probable equation. By this procedure, good food sources absorb more onlooker bees comparing with bad food sources. Each one of the onlooker bees, after finding a food source, will look for certain cycle in its neighborhood (limit), find a food source and evaluate its competency. The best food source which is located neighboring the source i and source i, itself, will be considered as new place of source $i$ and if the competency does not improve then it becomes a scout bee and looks for a new food source completely random. This is equal to random new source allocation (answers) to scout bees and becoming them to employed bees. After determining new location of each food source, the iteration of other $\mathrm{ABC}$ algorithm will be begun. This process is repeated again and again until it obtains the termination conditions [16, 17, 18 \& 19]. Food source neighboring a special source is gained by changing the amount of one of the parameters (aspects) of current answer and without trying other parameters. Assume that each answer includes $d$ parameter, $\mathrm{X}_{\mathrm{i}}=\left(\mathrm{x}_{\mathrm{i} 1}, \mathrm{X}_{\mathrm{i} 2} \ldots \mathrm{X}_{\mathrm{id}}\right)$, to determine an answer near $\mathrm{X}_{\mathrm{i}}$, for example $\mathrm{X}_{\mathrm{i}}$, one of the parameters of $X_{i}$ answer, for example $\mathrm{j}$ and another answer for instance, , $X_{k}=\left(x_{k 1}, X_{k 2} \ldots x_{k d}\right)$ were selected random. Except selected parameter of $\mathrm{j}$, all other $\mathrm{X}_{\mathrm{i}}^{\prime}$ parameters are same as $\mathrm{X}_{\mathrm{i}}, \mathrm{X}_{\mathrm{i}}^{\prime}=\left(\mathrm{x}_{\mathrm{i} 1}, \mathrm{X}_{\mathrm{i} 2}, \ldots, \mathrm{X}_{\mathrm{i}(\mathrm{j}-1)}\right)$, $\left.\mathrm{x}_{\mathrm{ij}}, \mathrm{x}_{\mathrm{i}(\mathrm{j}+1), \ldots \mathrm{x}} \mathrm{id}\right)$.

Parameter $\mathrm{j}$ is calculated from $\mathrm{X}_{\mathrm{i}}^{\prime}$ using equation [3] 
A. Ghorbannia Delavar, S. Hoseyny, R. Maghsoudi / TJ MCS Vol .5 No. 2 (2012) 105-114

$\mathrm{u}$ is a continuous random variable in range of $[-1,1]$. If the result passes over the acceptable range for parameter $j$, it arrange by the maximum of range [19]. In the following figure, artificial bee colony algorithm process is shown in Pseudo Code:

1: Initialize

2: REPEAT.

3: Move the employed bees onto their food source and evaluate the fitness

4: Move the onlookers onto the food source and evaluate their fitness

5: Move the scouts for searching new food source

6: Memorize the best food source found so far

7: UNTIL (termination criteria satisfied)

\section{Proposed Routing Algorithm}

Our proposed strategy to find optimized route based on QOS essentials, observing ABC swarm intelligence is presented in a diagram. Before surveying the following diagram, it is notable that we consider each link as one food source which has 5 dimensions including delay, cost, jitter, packet loss ratio and process time. The goal is to find an optimized route from edges which reflect the efficiency criterion of QOS parameter.

\subsection{Fitness Function}

Fitness function in this research is a more optimized version of which proposed in article [6]. Fitness function defines as each (series of) link (s) - means a route in network - , however, we add the $5^{\text {th }}$ constraint to increase accuracy of package delivery.

Total Time $=$ Process Time $+[$ Sending Time + Receiving Time $]$

We have:

Process Time $=$ Total Time $-[$ Sending Time + Receiving Time $]$

So:

Fitness $(\mathrm{p})=\mathrm{w}_{1} * \mathrm{e}^{-\operatorname{cost}(\mathrm{p})}+\mathrm{w}_{2} * \mathrm{e}^{-\operatorname{delay}(\mathrm{p})}+\mathrm{w}_{3} * \mathrm{e}^{-\mathrm{jitter}(\mathrm{p})}+\mathrm{w}_{4} * \mathrm{e}^{-\operatorname{loss}(\mathrm{p})} \quad[4]$

$+W_{5} * e^{\tau_{T}-\left(\tau_{R}+\tau_{A}\right)}$

In which $\tau_{T}, \tau_{R}$ and $\tau_{A}$ are total time, sending time and Receiving time, respectively.

\subsection{Initial Population}

To create initial population or initial food source we use data stimulated by Opnet software. The initial population includes $\mathrm{n}$ (number of edges) individuals, as mentioned before, each individual has 5 dimensions and each dimension is one of five mentioned parameters in fitness function.

\subsection{Proposed Strategy Diagram}


A. Ghorbannia Delavar, S. Hoseyny, R. Maghsoudi / TJ MCS Vol .5 No. 2 (2012) 105-114

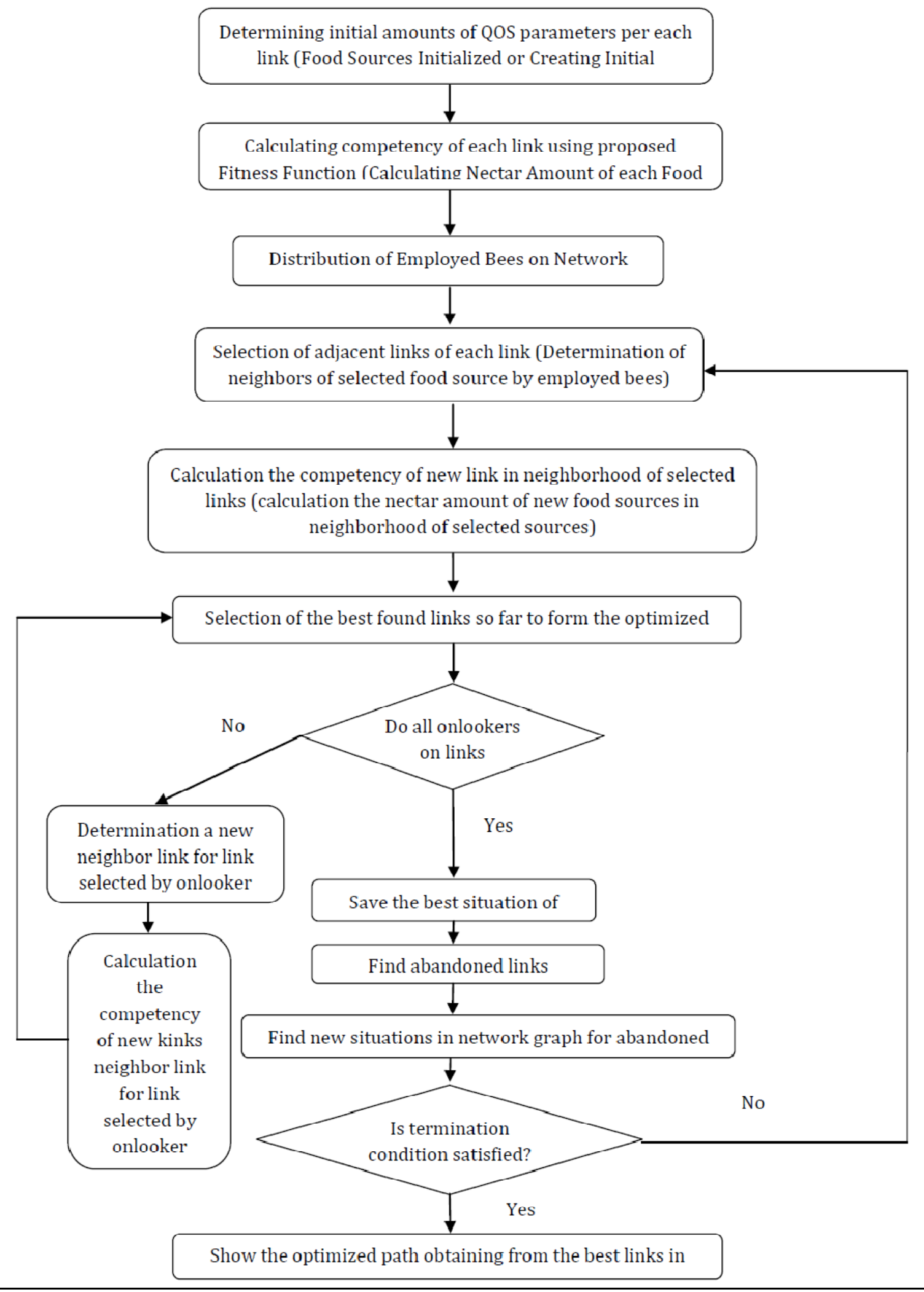

Figure 1. Proposed Routing Algorithm Diagram

\section{Experiment Results and Evaluation}

The main issue in testing chapter is to place the efficiency of proposed algorithm providing a path with optimized QOS and satisfaction of service essentials for multimedia applications. For 
more clear comparison, we concentrate on numerical aspects of quality such as throughput, point to point delay and execution time. Stimulations lead by powerful Opnet Modeler 14.5 and MATLAB Engineering Software. Using parameters in stimulation by Opnet are mentioned in Table 1.

Table 1. Simulation Parameters of Proposed Algorithm

\begin{tabular}{|c|c|}
\hline Number of Nodes (Edges) & 200 \\
\hline Type of Node & Ethcoax_station \\
\hline Type of Link & Eth_coax \\
\hline Transfer Protocol & UDP \& TCP \\
\hline Type of Service & Video Conference \\
\hline Stimulation Time & 250 Seconds \\
\hline
\end{tabular}

Figure 2 show the execution time of proposed algorithm comparing with current genetic algorithm which mentioned in Chapter 1. As mentioned in this figure, GA needs more time than proposed algorithm based on honey bee to execute. One of the reasons of QOS routing development based on $\mathrm{ABC}$ is the high - speed convergence of artificial bee colony in searching area which has seen clearly in figure 2 of this research. Figure 3 illustrates the efficiency comparison between proposed algorithm and current GA with video conference service. The diagram shows that throughput of proposed algorithm is very higher than GA but delivery delay and process time are less. Therefore it could be expressed that proposed method has better function and efficiency than traditional QOS routing versions.

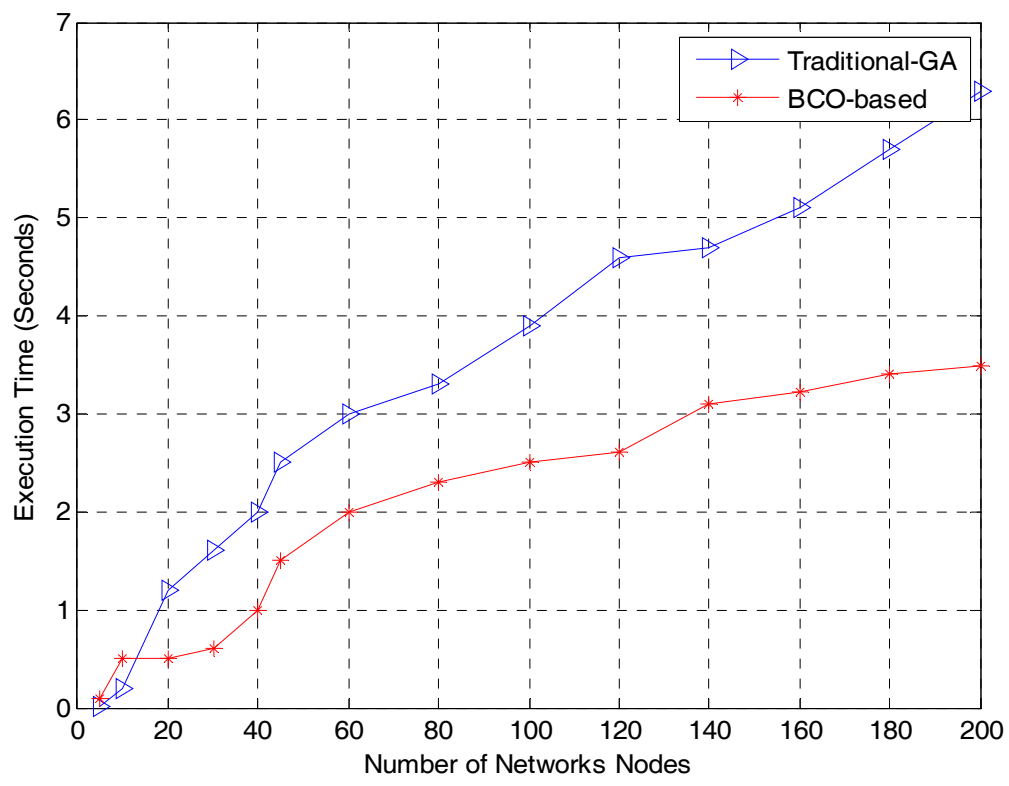

Figure 2. Comparison of Two Algorithms Execution 
A. Ghorbannia Delavar, S. Hoseyny, R. Maghsoudi / TJ MCS Vol .5 No. 2 (2012) 105-114

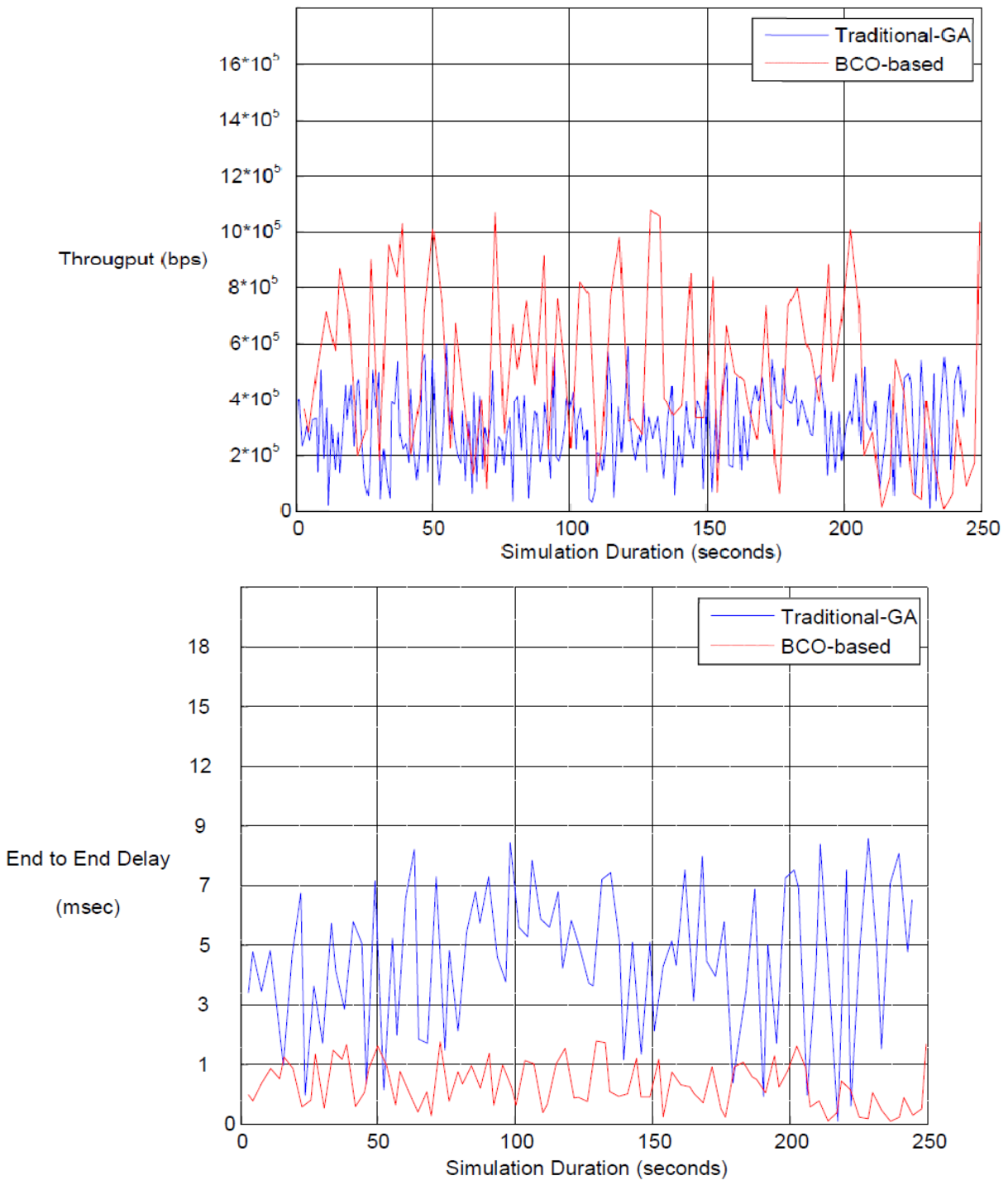

Figure 3. Efficiency Comparison of Two Methods using Video Conference Service

\section{Discussion and Conclusion}

In this article, at first we analyzed the intelligent algorithms which used to QOS optimized routing such as GA and ACO, then we proposed an optimized routing method based on swarm intelligence algorithm of artificial bee colony which increases convergence and diversity, observing the nature of this colony. We reached some approaches by theoretical analysis; 1 ) the proposed method shall have less execution time than typical genetic methods, 2) regarding richer features of searching in artificial bee colony, the more efficiency is expected, which we reached these results based on performed stimulations. It means that results of stimulation output shows better function of proposed algorithm as well in point to point delay and throughput. 


\section{A. Ghorbannia Delavar, S. Hoseyny, R. Maghsoudi / TJ MCS Vol .5 No. 2 (2012) 105-114}

\section{References.}

[1] Ping Chen, Tian-lin Dong, A fuzzy genetic algorithm for QoS multicast routing, Computer Communications 26 (2003) 506-512.

[2] A.T. Haghighat, K. Faez, M. Dehghan, A. Mowlaei, Y. Ghahremani, GA-Based Heuristic Algorithms for QoS Based Multicast Routing, Knowledge-Based Systems 16 (2003) 305-312.

[3] Jun Huang, Yanbing Liu, MOEAQ: A QoS-Aware Multicast Routing algorithm for MANET, Expert Systems with Applications 37 (2010) 1391-1399.

[4] Xingwei Wang, Jiannong Cao, Hui Cheng, Min Huang, QoS multicast routing for multimedia group communications using intelligent computational methods, Computer Communications 29 (2006) 2217-2229.

[5] F. Xiang, L. Junzhou, W. Jieyi, G. Guanqun, QoS routing based on genetic algorithm, Computer Communications 22 (1999) 1392-1399.

[6] Hua Wang, Zhao Shi, Anfeng Ge, Chaoying Yu, An optimized ant colony algorithm based on the gradual changing orientation factor for multi-constraint QoS routing, Computer Communications 32 (2009) 586-593.

[7] Muhammad Saleem, Gianni A. Di Caro, Muddassar Farooq, Swarm intelligence based routing protocol for wireless sensor networks: Survey and future directions, Information Sciences xxx (2010) $\mathrm{xxx}-\mathrm{xxx}$.

[8] G.A. Di Caro, Ant Colony Optimization and Its Application to Adaptive Routing in Telecommunication Networks, Ph.D. Thesis, Faculté des Sciences Appliquées, Université Libre de Bruxelles (ULB), Brussels, Belgium, 2004.

[9] G.A. Di Caro, F. Ducatelle, L. Gambardella, AntHocNet: an adaptive nature-inspired algorithm for routing in mobile ad hoc networks, European Transactions on Telecommunications (ETT) 16 (2) (2005) 443-455 (Special Issue on Self Organization in Mobile Networking).

[10] G.A. Di Caro, F. Ducatelle, L. Gambardella, Theory and practice of Ant Colony Optimization for routing in dynamic telecommunications networks, in: $\mathrm{N}$.

Sala, F. Orsucci (Eds.), Reflecting Interfaces: The Complex Coevolution of Information Technology Ecosystems, Idea Group, Hershey, PA, USA, 2008, pp.

$11-32$.

[11] R. Ghasemaghaei, M.A. Rahman, W. Gueaieb, A. El Saddik, Ant colony-based reinforcement learning algorithm for routing in wireless sensor networks,

in: Proceedings of the IEEE Instrumentation and Measurement Technology Conference (IMTC), 2007, pp. 2173-2178.

[12] K.M. Sim, W.H. Sun, Ant colony optimization for routing and load balancing: survey and new directions, IEEE Transactions on System, Man and Cybernetics 33 (5) (2003) 560-572.

[13] Yannis Marinakis, Magdalene Marinaki, Georgios Dounias, Honey bees mating optimization algorithm for the Euclidean traveling salesman problem, Information Sciences xxx (2010) xxx-xxx.

[14] M. Farooq, G.A. Di Caro, Routing protocols inspired by insect societies, in: C. Blum, D. Merkle (Eds.), Swarm Intelligence, Introduction and Applications,

Natural Computing Series, Springer-Verlag, 2008, pp. 101-160. 
A. Ghorbannia Delavar, S. Hoseyny, R. Maghsoudi / TJ MCS Vol .5 No. 2 (2012) 105-114

[15] M. Saleem, M. Farooq, Beesensor: a bee-inspired power aware routing protocol for wireless sensor networks, in: Proceedings of the 4th EvoCOMNET

Workshop, LNCS, vol. 4448, 2007.

[16] Smart L.Sabat, Siba K.Udgata, Ajith Abraham., 2010. Artificial bee colony algorithm for small signal model parameter extraction of MESFET, Engineering Application of Artificial Intelligence 23 (5), $689-694$.

[17] Dervis Karaboga, Bahriye Akay, A Comparative Study of Artificial Bee Colony Algorithm, Applied Mathematics and Computation 214(2009) 108-132.

[18] Shyam Sundar, Alok Singh, A Swarm Intelligence Approach to the Quadratic Minimum Spanning Tree Problem, Information sciences 180(2010) 3182-3191.

[19] Bahriye Akay, Dervis Karaboga, A Modified Artificial Bee Colony Algorithm for Real-parameter Optimization, Information Sciences, Available online 27 July 2010.

[20] Li-Pei Wong, Chin Soon Chong, An Efficient Bee Colony Optimization Algorithm for Traveling Salesman Problem using Frequency-based Pruning, 2009 7th IEEE International Conference on Industrial Informatics (INDIN 2009). 